\title{
Change Detection Method with Multi-temporal Satellite Images based on Wavelet Decomposition and Tiling
}

\author{
Kohei Arai \\ Saga University \\ Faculty of Science and Engineering \\ Saga City, Japan
}

\begin{abstract}
Change detection method with multi-temporal satellite images based on Wavelet decomposition with Daubechies wavelet function (Multi Resolution Analysis), and tiling is proposed. The method allows detection of changes in time series analysis and is not sensitive to geometric distortions included in the satellite images. In this paper, the author proposed a method based on MRA as a method for extracting change points from satellite images acquired over many periods. Change detection method with multi-temporal satellite images based on Wavelet decomposition and tiling is proposed. The method allows to detect changes and is not sensitive to geometric distortions included in the satellite images. The experimental results with simulation image and a Landsat Thematic Mapper (TM) image show that more appropriate changes can be detected with the proposed method in comparison with the existing method of subtraction. When applied to simulations and real satellite images, it was confirmed that they were robust to minute nonlinear geometric distortion.
\end{abstract}

Keywords-Daubechies wavelet; multi-resolution analysis: MRA; change detection; multi-temporal satellite image; geometric distortion; Landsat Thematic Mapper (TM) image

\section{INTRODUCTION}

Change detection is important for time series analysis obviously. Trend analysis of the global warming issues is needed for identifying the locations and the timing for severely damaged areas and timing for instance. From the time series of satellite-based imagery data, it is possible to check the location and the timing of which warming phenomena is getting severe due to the estimated carbon dioxide and methane as well as nitric acid concentrations based on change detections.

As an example of applying wavelet analysis (development, transformation, etc.) to processing and analysis of earth observation satellite images, a method of superimposing multiple visible images after wavelet transformation [1], superimposing multiple Synthetic Aperture Radar: SAR images with different off-nadir angles After the wavelet transform [2], the method of applying the wavelet transform to the pattern of the annual fluctuation of the sea surface temperature estimated from the satellite data to extract its features [3], and the wavelet transform to the extraction of the surface roughness of sea ice [4], and [5] a method of extracting spatial features from images from which soil moisture has been extracted.
Extraction of water mass features from satellite images using polar coordinate representation Wavelet is discussed [6].

In this paper, the author examines a method of extracting change points of satellite images acquired over many periods using multi-resolution analysis (MRA). Extraction of change points from satellite image data acquired at each time as a method of performing the above, a method of taking a difference between images can be considered. However, the satellite image includes geometric distortion, and pixels resulting from the distortion are also extracted as change points in the difference image.

Among them, the linear distortion can be removed relatively easily, but the removal of the nonlinear distortion is not easy. Therefore, the author applied a multi-resolution analysis to the satellite image and devised a method of extracting a change point robust to nonlinear distortion by reducing the number of nodes, and confirmed the effect using a simulation image and a satellite image. The author reports here because good results were obtained.

In the following section, related research works and research background including motivation of the research are described. Then, the proposed context classification method is described followed by experimental method together with experimental results. After that, concluding remarks and some discussions are described.

\section{RELATED RESEARCH WORKS}

Improved method of change detection method for remotely sensed images is proposed [7]. On the other hand, $\mathrm{CO}_{2}$ concentration changes detection in time and space domains by means of wavelet analysis of MRA: Multi Resolution Analysis is proposed [8]. Method for support length determination of base function of Wavelet for edge and line detection as well as moving object and change detections is proposed [9].

Wavelet based change detection for four-dimensional assimilation data in space and time domains is also proposed [10]. Meanwhile, method for psychological status monitoring with line-of-sight vector changes (Human eyes movements) detected with wearing glass is proposed [11].

Method for real time text extraction of digital manga comic is proposed [12]. On the other hand, extraction of line features from multifidus muscle of Computer Tomography: CT scanned 
images with morphological filter together with wavelet multi resolution analysis is proposed [13]. Method for extraction product information from TV commercial is also proposed [14].

Text extraction from TV commercial using blob extraction method is proposed [15]. Eye-based human-computer interaction allowing phoning, reading e-book/e-comic/elearning, Internet browsing, and TV information extraction is also proposed [16]. Meanwhile, comparative study of feature extraction components for several wavelet transformations for ornamental plants is conducted [17].

Human gait gender classification using 3D discrete wavelet transformation feature extraction is proposed [18]. Comparison contour extraction based on layered structure and Fourier descriptor on image retrieval is conducted [19]. On the other hand, phytoplankton discrimination method with wavelet descriptor-based shape feature extraction from microscopic images is proposed [20].

\section{RESEARCH BACKGROUND}

\section{A. Wavelet Transformation}

The Wavelet transformation of the function $f(x)$ by the mother wavelet $\psi(x)$ is given by the following equation.

$\left(W_{\varphi} f\right)(b, a)=\sqrt{\frac{1}{|a|}} \int_{-\infty}^{\infty} \varphi\left(\frac{x-b}{a}\right) f(x) d x$

Note that $\psi(\bullet)$ is the complex conjugate of $\psi(\bullet)$. In this paper, Haar mother wavelet is used.

\section{B. Haar Wavelet Function}

Haar wavelet is one of the Wavelets. In 1909, Alfréd Haar announced it under the name of the Haar train. It is also one of the Daubechies wavelets. Haar Wavelets are the simplest wavelets. The disadvantage is that they are not continuous and therefore not differentiable.

The definition of the Wavelet transformation is as follows:

$\psi(t)=\{1$ for $0 \leq t<1 / 2$,

-1 for $1 / 2 \leq t<1$,

0 for otherwise.

The corresponding scaling functions are:

$\phi(t)=\{1$ for $0 \leq t<1$, 0 otherwise.

Haar Wavelet function is shown in Fig. 1. $\psi(t)$

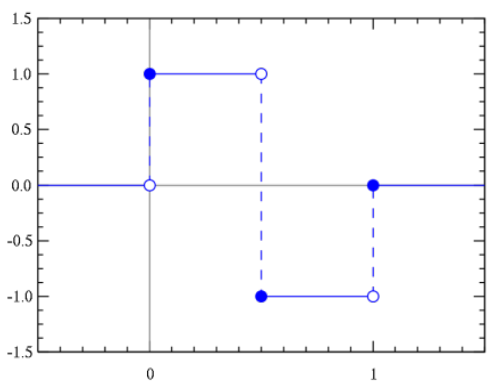

Fig. 1. Haar Wavelet Function.

\section{Duabechies Wavelet Function}

Fig. 2 shows Daubechies 4 tap Wavelet functions (red line indicates Wavelet function while blue line shows scaling function).

\section{2D(Two Dimensional) Discrete Wavelet Transformation}

For 2D image signals, this process is performed horizontally and vertically one level at a time. Fig. 3 shows the band components when two-dimensional DWT is performed twice.

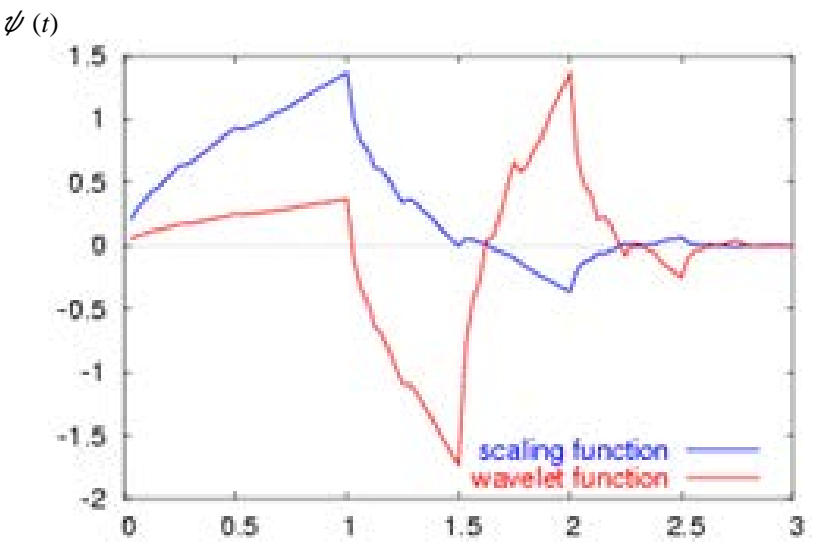

Fig. 2. Daubechies 4 tap Wavelet.

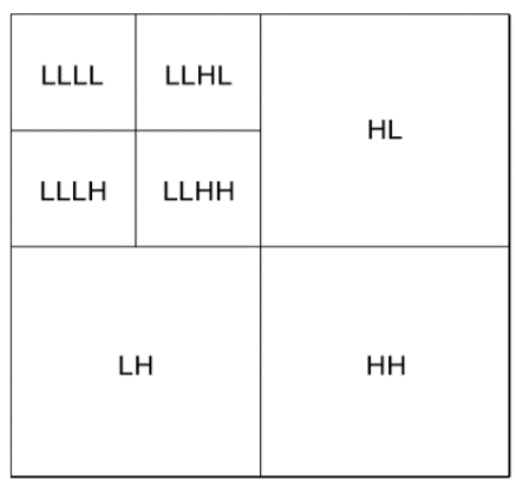

Fig. 3. Band Components after the 2D DWT.

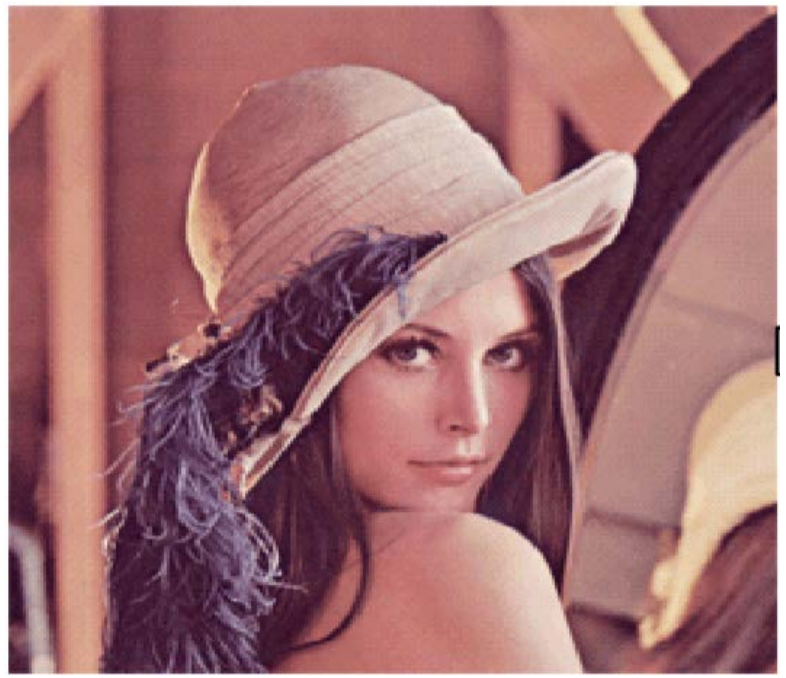

(a) Original Image of "Lena” Included in SIDBA Database 


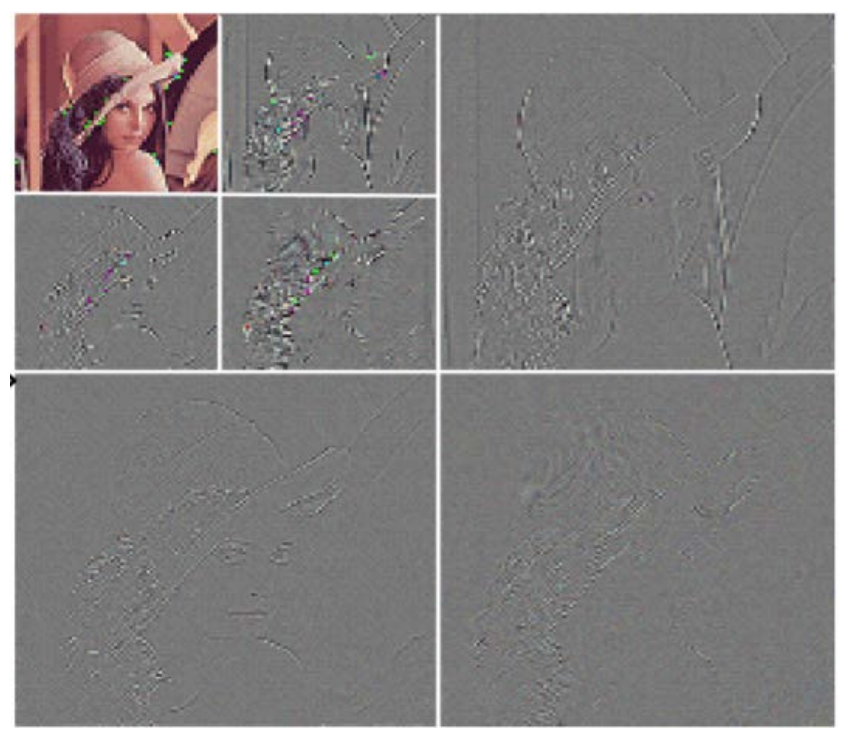

(b) Resultant Image of 2D DWT

Fig. 4. Example of 2D DWT of the Image of "Lena" in the Image Database of SIDBA.

In the figure, $\mathrm{L}$ indicates a low frequency component, and $\mathrm{H}$ indicates a high frequency component. The image is decomposed into four bands (LL, LH, HL, HH) by the first two-dimensional DWT, and the lowest band component (LL) is further divided into four bands (LLLL, LLLH, LLHL, LLHH). Fig. 4 shows the results of two-dimensional DWT performed twice on an actual image.

\section{E. Tiling}

Tiling divides an image into several blocks (tiles), and then treats the tiles as one independent image. The size of the tile is arbitrarily selectable, and its minimum unit is $1 \times 1$.

\section{Proposed MEthod}

Change detection by Multi-Resolution Analysis: MRA is proposed. When MRA is applied to multi-temporal satellite images, it is divided into four components: LL, LH, HL, and HH. Since the image of the LL component does not reflect minute non-linear geometric distortion, it can be expected to be robust against such distortion.

The LL component represents a global feature of the image before the wavelet transform is performed, and the difference between the LL components of the images acquired at many times is considered to represent a change point. At this time, the setting of the number of levels of the MRA uses a difference between the number of points considered as the change points before conversion and the number of points considered as the change points after conversion.

\section{EXPERIMENT}

\section{A. Simulation Study}

Simulation images were generated, and the validity of the proposed method was evaluated. With respect to the original image (the left figure in Fig. 5), a case of a changing image (the left figure of Fig. 6) and a case of a distorted image (the right figure of Fig. 6) were examined.

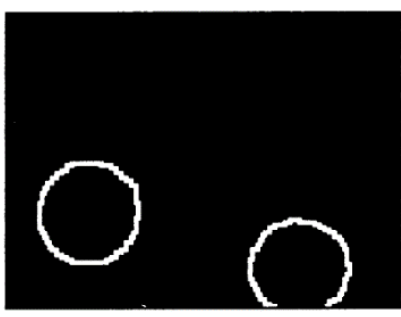

(a) Original simulation image

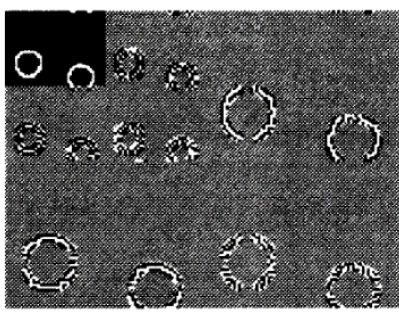

(b) After 2D DWT
Fig. 5. Original Simulation Image and the Resultant Image after the 2D DWT.
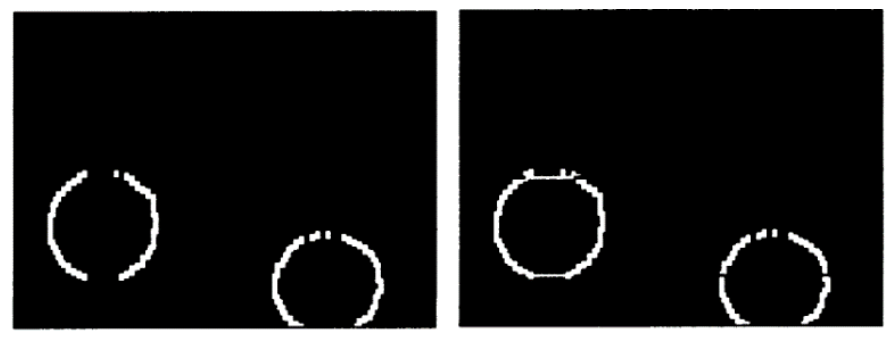

Fig. 6. Non-Distorted [Left] and Distorted [Right] Images.

The right diagram in Fig. 5 is an image obtained by performing the Daubechies wavelet transform on the left diagram in Fig. 5. The left diagram in Fig. 7 shows the difference between the left diagram in Fig. 5 and the left diagram in Fig. 6 by the wavelet transform. This is the result of direct extraction without performing. The right diagram of Fig. 7 is a result of directly extracting the difference between the right diagram of Fig. 5 and the right diagram of Fig. 6 without performing the wavelet transform. The right diagram in Fig. 8 is an image obtained by performing a Daubechies wavelet transform on the right diagram in Fig. 8. The left diagram in Fig. 9 is an image showing the difference between the right diagram in Fig. 5 and the left diagram in Fig. 6. The right diagram in Fig. 9 is an image showing the difference between the right diagram in Fig. 5 and the right diagram in Fig. 6. The white areas in Fig. 7 and Fig. 9 represent the change points.
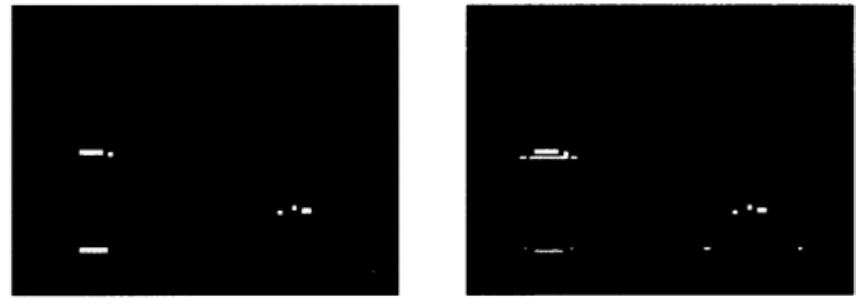

Fig. 7. Difference Images between the Original and the Changed [left] and the Distorted [Right] Images.
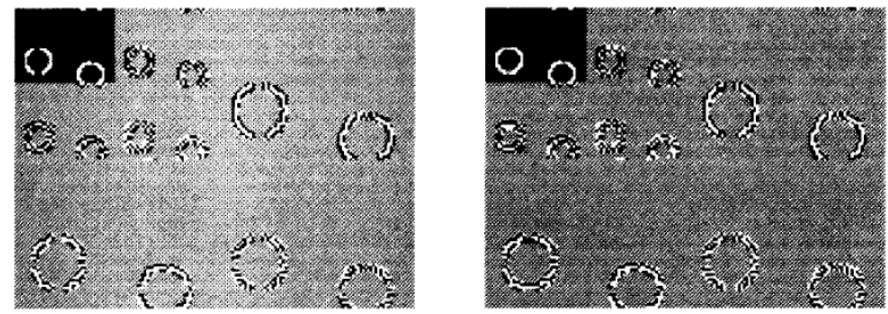

Fig. 8. The Resultant Images of MRA with 2D Daubechies Wavelet Transformation for the Changed [left] and the Distorted [Right] Images. 

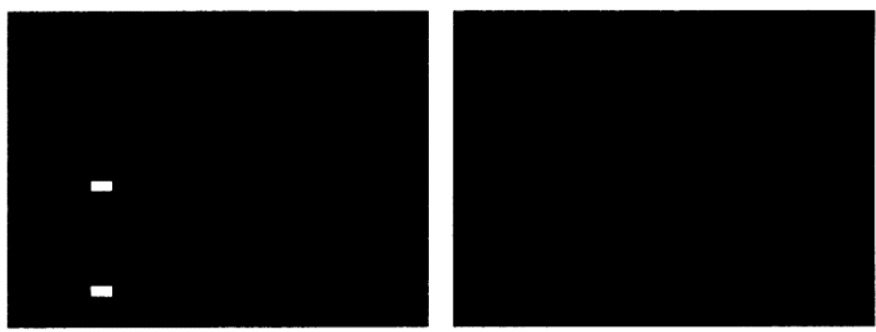

Fig. 9. Difference Images between the Original and the Changed [left] and the Distorted [right] Images after the MRA.

\section{B. Experiment with Actual Satellite Image}

In addition, experiments using satellite images were also conducted. The data used this time is Landsat TM (Thematic Mapper) images acquired at many times in Tokyo Bay's waterfront subcenter. TM images are used for creating land cover maps. Fig. 10 and 11 show the acquired TM images. By performing the change extraction, it is possible to easily grasp the progress of the development of the seaside subcenter.

Fig. 12 shows the result of directly extracting the difference between Fig. 10 and Fig. 11 without performing the wavelet transform. Fig. 13 is an image obtained by performing the Daubechies wavelet transform on the image of Fig. 10 and Fig. 14 is an image obtained by performing the Daubechies wavelet transform on the image of Fig. 12. Fig. 15 is an image showing the difference between Fig. 13 and Fig. 14. The white areas in Fig. 12 and 15 represent the changing points.

When the difference is directly extracted without performing the wavelet transform, even a minute nonlinear geometric distortion is extracted as a change point. On the other hand, when the differences are extracted using the wavelet transform, it is understood that they are not extracted as the change points. Therefore, it can be said that the change point extraction method using the wavelet transform is robust to minute nonlinear geometric distortion as a method for extracting change points from satellite data acquired at many times.

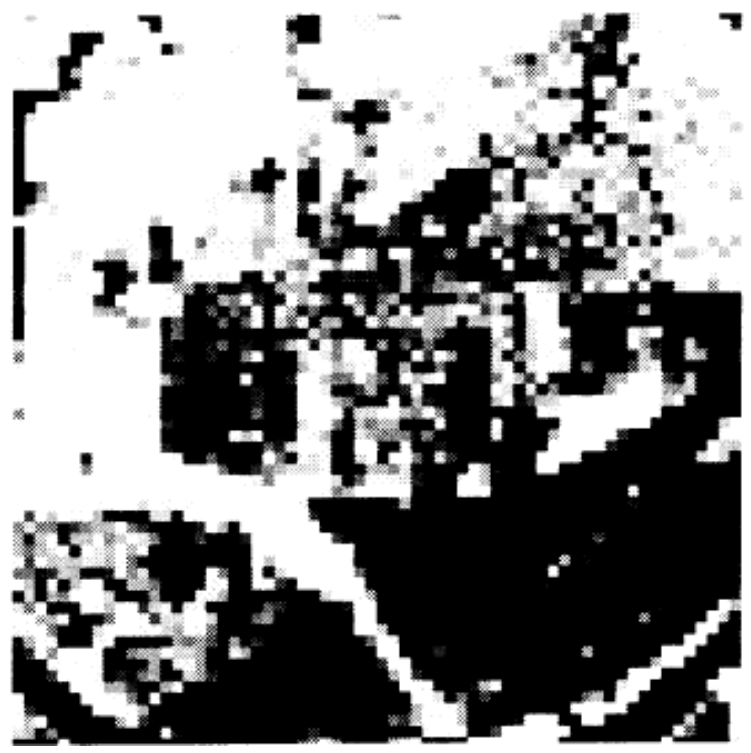

Fig. 10. Landsat TM Image before Change.

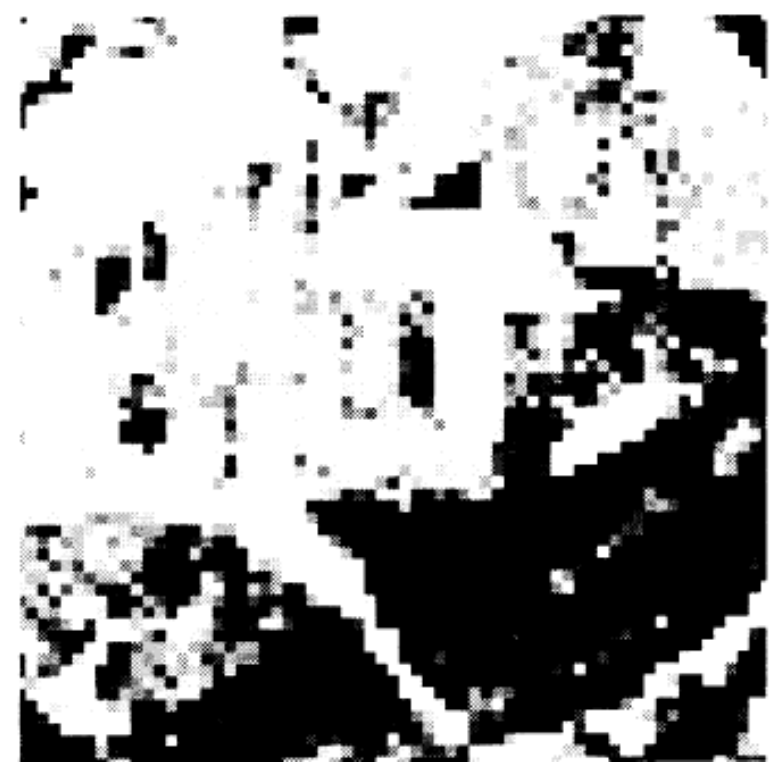

Fig. 11. Landsat TM Image after Change.

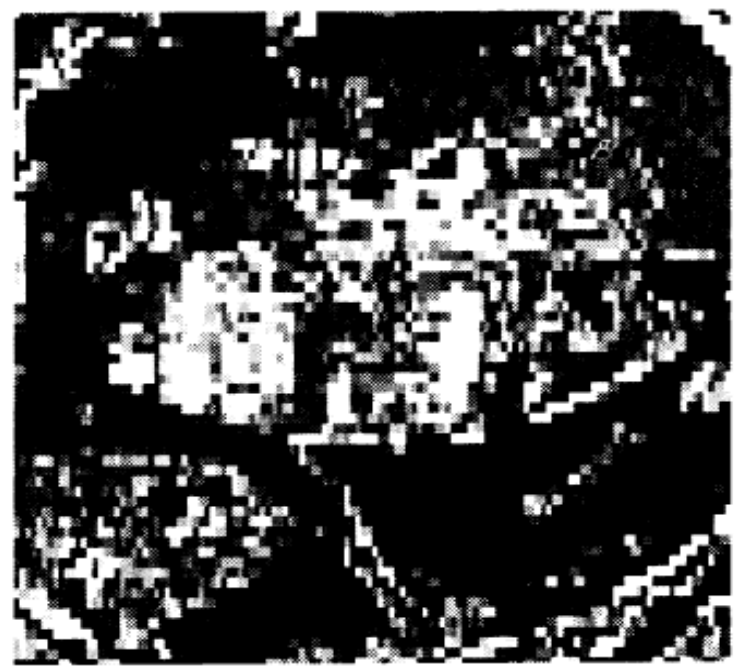

Fig. 12. A Difference Image between before and after Change.

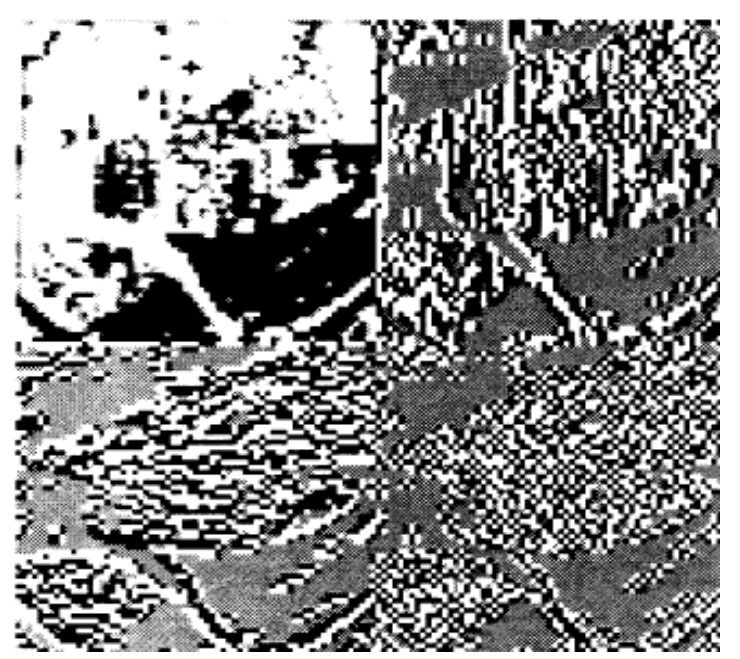

Fig. 13. The Resultant Image of MRA with 2D Daubechies Wavelet Transformation [Before Change Image]. 


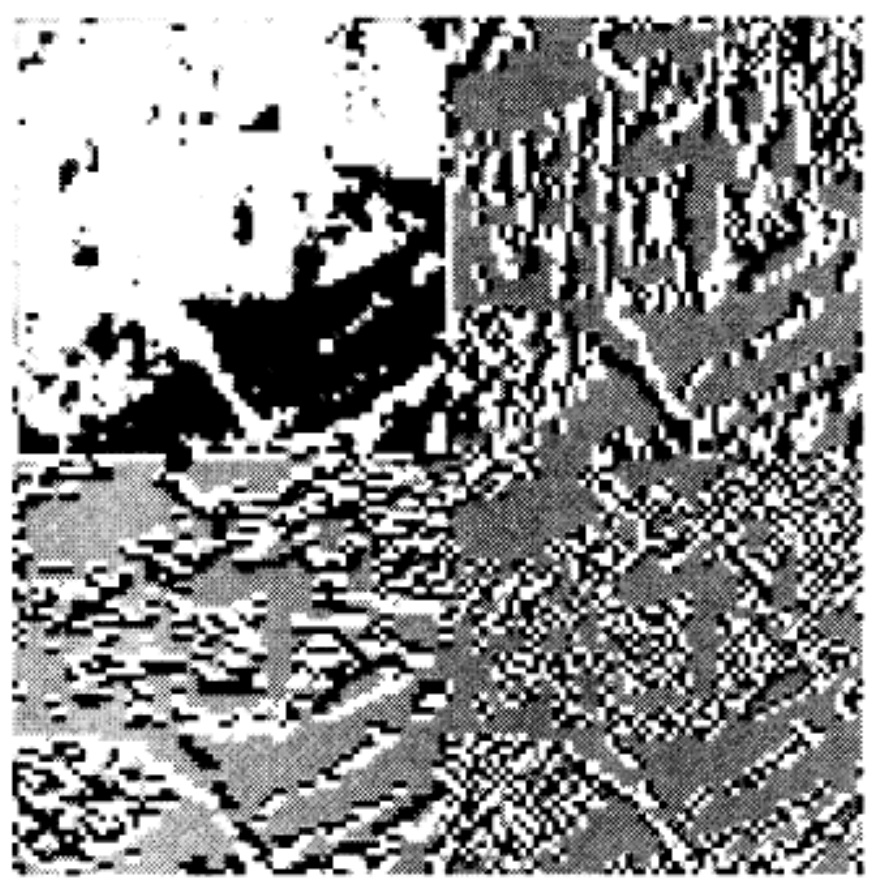

Fig. 14. The Resultant Image of MRA with 2D Daubechies Wavelet Transformation [After Change Image].

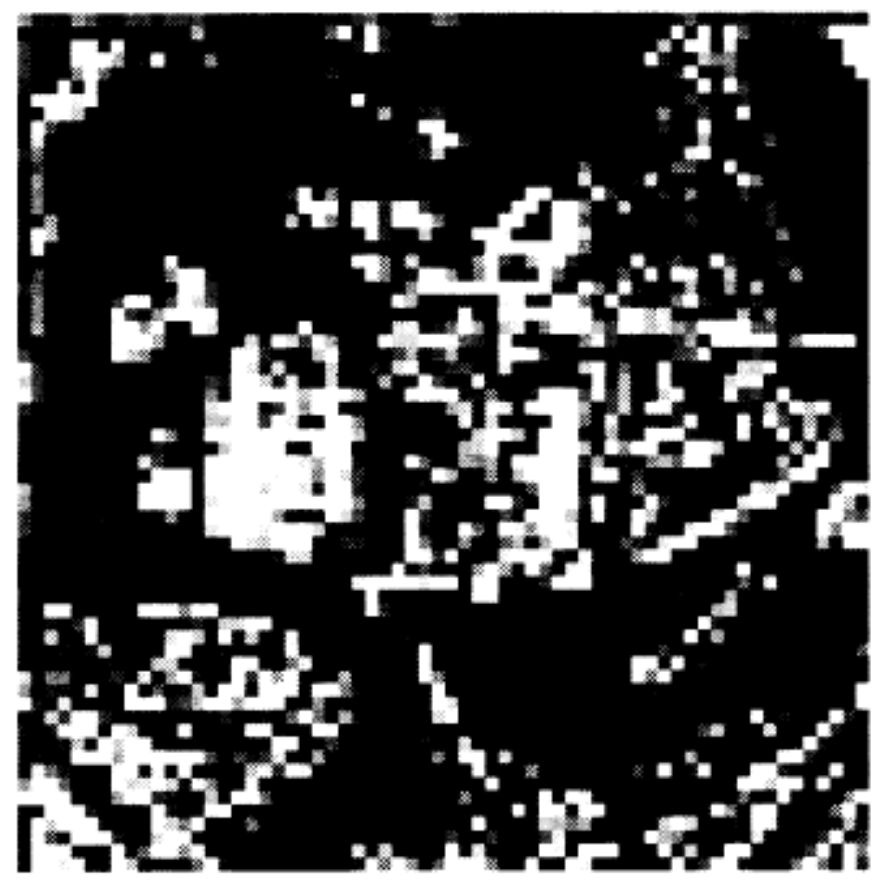

Fig. 15. A Difference Image between Before and After Change through MRA.

\section{CONCLUSION}

In this paper, the author proposed a method based on MRA as a method for extracting change points from satellite images acquired over many periods. Change detection method with multi-temporal satellite images based on Wavelet decomposition and tiling is proposed. The method allows detecting changes and is not sensitive to geometric distortions included in the satellite images. The experimental results with simulation image and a Landsat Thematic Mapper (TM) image show that more appropriate changes can be detected with the proposed method in comparison with the existing method of subtraction.

When applied to simulations and real satellite images, it was confirmed that they were robust to minute nonlinear geometric distortion.

\section{FUTURE RESEARCH WORKS}

The proposed method is adopted in the real earth observation satellite imagery data, and it is a future subject to realize a more usable change detection method. In the future, the author will compare with conventional methods such as relaxation method. The author also considers the definition of Wavelet transform on polar coordinates and its application to water mass extraction.

\section{ACKNOWLEDGMENT}

The author would like to thank Dr. Kaname Seto of former student of Saga University and Dr. Leland M. Jameson of Naval Research Laboratory for their contribution of this study. The author, also, would like to thank Professor Dr. Hiroshi Okumura and Professor Dr. Osamu Fukuda for their valuable discussions.

\section{REFERENCES}

[1] Jean-Pierre Djamdji, Albert Bijaoui, Roger Maniere: "Geometrical Registration of Images: The Multiresolution Approach," Photogrammetric Engineering \& Remote Sensing, Vol.59, No. 5, pp.645-653, (1993).

[2] Wooil M. Moon, J.S.Won, Vern Singhroy. and Paul D. Lowman Jr.: "ERS-1 and CCRS C-SAR data integration for lookdirection bias correction using wavelet transform," Canadian Journal of Remote Sensing, Vol.20, No.3, pp.280-285, (1994).

[3] Nankin Mak: "Orthogonal Wavelet analysis: Interannual Variability in Sea Surface Temperature," Bulletin of the American Meteorological Society, Vol.76, No.11, pp.2179-2186, (1995).

[4] Ronald W. Lindsay, Donald B. Percival. and D. Andrew Rothrock: "The Discrete Wavelet Transform and the Scale Analysis of the Surface Properties of Sea Ice," IEEE Transactions on Geoscience and Remote Sensing, Vol. 34, No.3, pp.771-787, (1996).

[5] Zhenglin Hu, Yizong Chen, and Shafiqul Islam: "Multiscaling properties of soil moisture images and decomposition of largeand small-scale features using wavelet transforms," International Journal of Remote Sensing, Vol.19, No.13, pp.2451-2467, (1998).

[6] Kohei Arai, Kaname Seto, Leland M. Jameson: "Extraction of water mass features from satellite images using polar coordinate representation Wavelet", Journal of Japan Society for Visualization Information, Vol.19, Suppl.1, No.1, pp.99-102, Kogakuin University, (1999).

[7] H. Okumura and Kohei Arai, Improvement of change detection method for remotely sensed images, Proceedings of SPIE European Remote Sensing, Invited Paper, ERS-10-RS07-61, 2010.

[8] Kohei Arai, CO2 concentration change detection in time and space domains by means of wavelet analysis of MRA: Multi Resolution Analysis, International Journal of Advanced Computer Science and Applications, 2, 8, 82-86, 2011.

[9] Kohei Arai, Method for support length determination of base function of wavelet for edge and line detection as well as moving object and change detections, International Journal of Research and Reviews on Computer Science, 2, 4, 1133-1139, 2011.

[10] Kohei Arai, Wavelet based change detection for four dimensional assimilation data in space and time domains, International Journal of Advanced Computer Science and Applications, 3, 11, 71-75, 2012.

[11] Kohei Arai, Kiyoshi Hasegawa, Method for psychological status monitoring with line of sight vector changes (Human eyes movements) 
detected with wearing glass, International Journal of Advanced Research in Artificial Intelligence, 2, 6, 65-70, 2013.

[12] Kohei Arai, Tolle Herman, Method for real time text extraction of digital manga comic, International Journal of Image Processing, 4, 6, 669-676, 2011

[13] Kohei Arai, Yuichiro Eguchi and Yoichiro Kitajima, Extraction of line features from multifidus muscle of CT scanned images with morphological filter together with wavelet multi resolution analysis, International Journal of Advanced Computer Science and Applications, 2, 8, 60-66, 2011.

[14] Kohei Arai and Tolle Herman, Method for extraction product information from TV commercial, International Journal of Advanced Computer Science and Applications, 2, 8, 125-131, 2011.

[15] Kohei Arai and Tolle Herman, Text extraction from TV commercial using blob extraction method. International Journal of Research and Review of Computer Science, 2, 3, 895-899, 2011.

[16] Kohei Arai, Ronny Mardiyanto, Eye-based human-computer interaction allowing phoning, reading e-book/e-comic/e-learning, Internet browsing and TV information extraction, International Journal of Advanced Computer Science and Applications, 2, 12, 26-32, 2011.

[17] Kohei Arai, Indra Nugraha Abdullar, H.Okumura, Comparative study of feature extraction components for several wavelet transformations for ornamental plants, International Journal of Advanced Research in Artificial Intelligence, 3, 2, 5-11, 2014.

[18] Kohei Arai, Rosa Andrie Asmara, Human gait gender classification using 3D discrete wavelet transformation feature extraction, International Journal of Advanced Research in Artificial Intelligence, 3, 2, 12-17, 2014.
[19] Cahya Rahmad, Kohei Arai, Comparison contour extraction based on layered structure and Fourier descriptor on image retrieval, International Journal of Advanced Computer Science and Applications, 6, 12, 71-74, 2015.

[20] Kohei Arai, Phytoplankton discrimination method with wavelet descriptor based shape feature extraction from microscopic images, Journal of RIMS Signal and time frequency analysis, Kokyuroku edited by Ryuichi Ashino, Kyoto University, 50-86, 2010.

\section{AUTHOR's PROFILE}

Kohei Arai, He received BS, MS and PhD degrees in 1972, 1974 and 1982, respectively. He was with The Institute for Industrial Science and Technology of the University of Tokyo from April 1974 to December 1978 also was with National Space Development Agency of Japan from January, 1979 to March, 1990. During from 1985 to 1987, he was with Canada Centre for Remote Sensing as a Post Doctoral Fellow of National Science and Engineering Research Council of Canada. He moved to Saga University as a Professor in Department of Information Science on April 1990. He was a councilor for the Aeronautics and Space related to the Technology Committee of the Ministry of Science and Technology during from 1998 to 2000 . He was a councilor of Saga University for 2002 and 2003. He also was an executive councilor for the Remote Sensing Society of Japan for 2003 to 2005. He is a Science Council of Japan Special Member since 2012. He is an Adjunct Professor of University of Arizona, USA since 1998. He also is Vice Chairman of the Science Commission "A" of ICSU/COSPAR since 2008 then he is now award committee member of ICSU/COSPAR. He wrote 55 books and published 620 journal papers as well as 450 conference papers. He received 66 of awards including ICSU/COSPAR Vikram Sarabhai Medal in 2016, and Science award of Ministry of Mister of Education of Japan in 2015. He is now Editor-in-Chief of IJACSA and IJISA.

http://teagis.ip.is.saga-u.ac.jp/index.html 